\title{
Co-tunneling current and shot noise in quantum dots
}

\author{
Axel Thielmann, ${ }^{1}$ Matthias H. Hettler, ${ }^{1}$ Jürgen König, ${ }^{2}$ and Gerd Schön ${ }^{1,3}$ \\ ${ }^{1}$ Forschungszentrum Karlsruhe, Institut für Nanotechnologie, 76021 Karlsruhe, Germany \\ ${ }^{2}$ Institut für Theoretische Physik III, Ruhr-Universität Bochum, 44780 Bochum, Germany \\ ${ }^{3}$ Institut für Theoretische Festkörperphysik, Universität Karlsruhe, 76128 Karlsruhe, Germany
}

(Dated: February 2, 2008)

\begin{abstract}
We derive general expressions for the current and shot noise, taking into account non-Markovian memory effects. In generalization of previous approaches our theory is valid for arbitrary Coulomb interaction and coupling strength and is applicable to quantum dots and more complex systems like molecules. A diagrammatic expansion up to second-order in the coupling strength, taking into account co-tunneling processes, allows for a study of transport in a regime relevant to many experiments. As an example, we consider a single-level quantum dot, focusing on the Coulombblockade regime. We find super-Poissonian shot noise due to spin-flip co-tunneling processes at an energy scale different from the one expected from first-order calculations, with a sensitive dependence on the coupling strength.
\end{abstract}

PACS numbers: 73.63.-b, 73.23.Hk, 72.70.+m

Introduction. - The study of shot noise in transport through mesoscopic devices, such as quantum dots or molecules, has become a field of intense theoretical and experimental research [1]. It provides additional information, not contained in the current, about system parameters that govern the electronic transport 2]. For weak coupling between the dot and metallic electrodes, transport is dominated by sequential tunneling, usually described by first-order perturbation theory in the coupling strength. Shot noise in this limit has been studied for a variety of models, including effects from electronic interaction [2, 3], multi-level dots [4, 5], inclusion of photonic and vibrational modes [5, [6], or spin-polarized leads 7]. However, a theory taking fully into account interaction effects as well as arbitrary coupling strength has not been presented. Expressions for the shot noise in terms of non-equilibrium Green functions have been derived only in the absence of Coulomb interactions 8$]$ or in a perturbative expansion thereof [9, 10].

Shot noise has been measured in various experimental realizations [11, 12, 13]. As the strength of the coupling in a given experiment is not a priori known, it is unclear whether first order calculations are sufficient. Second-order tunneling (co-tunneling [14]) processes can play an important role for the conductance 15], particularly in the Coulomb-blockade regime. Shot noise in this regime has been discussed in Ref. 16 where the possibility of super-Poissonian noise was suggested. However, that work was limited to pure co-tunneling processes, which is too restrictive for the experiment of Ref. 15.

In this Letter we provide a general formulation of the current and shot noise, valid for arbitrary coupling strength, while accounting fully for the Coulomb interaction. By making use of a diagrammatic formulation we expand the expressions up to second order in the coupling strength and study as an example a single-level quantum dot, which is out of equilibrium due to an applied bias voltage. The theory covers the Coulomb-blockade regime (low bias), the sequential tunneling regime (bias larger than the first single-charge excitation energy) and the crossover between both. We find super-Poissonian shot noise in the Coulomb-blockade regime due to spinflip co-tunneling processes at a different energy scale than expected from first-order calculations. Furthermore, we show that the noise to current ratio is highly sensitive to the coupling strength. This may serve as an additional spectroscopic tool for the couplings.

The model. - The Anderson impurity model is based on the Hamiltonian $\hat{H}=\hat{H}_{\mathrm{L}}+\hat{H}_{\mathrm{R}}+\hat{H}_{\text {dot }}+$ $\hat{H}_{\mathrm{T}, \mathrm{L}}+\hat{H}_{\mathrm{T}, \mathrm{R}}$ with $\hat{H}_{r}=\sum_{k \sigma} \epsilon_{k \sigma r} a_{k \sigma r}^{\dagger} a_{k \sigma r}, \hat{H}_{\mathrm{dot}}=$ $\sum_{\sigma} \epsilon_{\sigma} c_{\sigma}^{\dagger} c_{\sigma}+U n_{\uparrow} n_{\downarrow}$ and $\hat{H}_{\mathrm{T}, r}=\sum_{k \sigma}\left(t_{r} a_{k \sigma r}^{\dagger} c_{\sigma}+\right.$ h.c. $)$ for $r=\mathrm{L}, \mathrm{R}$. Here, $\hat{H}_{\mathrm{L}}$ and $\hat{H}_{\mathrm{R}}$ describe the left and right electrode with non-interacting electrons, $\hat{H}_{\mathrm{dot}}$ the quantum dot with one (spin-dependent) level of energy $\epsilon_{\sigma}$ and Coulomb interaction $U$ for double occupancy of the dot. Tunneling between the leads and the dot is modeled by $\hat{H}_{\mathrm{T}, \mathrm{L}}$ and $\hat{H}_{\mathrm{T}, \mathrm{R}}$. The coupling strength is characterized by the intrinsic line width $\Gamma_{r}=2 \pi \rho_{e}\left|t_{r}\right|^{2}$, and $\Gamma=\Gamma_{\mathrm{L}}+\Gamma_{\mathrm{R}}$, where $\rho_{e}$ is the (constant) density of states of the leads. The creation operators $a_{k \sigma r}^{\dagger}$ and $c_{\sigma}^{\dagger}$ refer to the electrodes and the impurity (dot), and $n_{\sigma}=c_{\sigma}^{\dagger} c_{\sigma}$.

We are interested in the current $I$ and the (zerofrequency) current noise $S$, which for $e V \gg k_{\mathrm{B}} T$ is dominated by shot noise. Both are related to the (symmetrized) current operator $\hat{I}=\left(\hat{I}_{\mathrm{R}}-\hat{I}_{\mathrm{L}}\right) / 2$ with $\hat{I}_{r}=$ $-i(e / \hbar) \sum_{k \sigma}\left(t_{r} a_{k \sigma r}^{\dagger} c_{\sigma}-h . c.\right)$, from which we obtain $I=\langle\hat{I}\rangle$ and

$$
S=2 \int_{-\infty}^{0} d t\left[\langle\hat{I}(t) \hat{I}(0)+\hat{I}(0) \hat{I}(t)\rangle-2\langle\hat{I}\rangle^{2}\right] .
$$

Diagrammatic technique. - In Ref. 2 we formulated a theory of current noise for transport to first order in $\Gamma$, based on a diagrammatic language that has been de- 
velopped for a systematic perturbation expansion of the current through localized levels 17]. All transport properties are governed by the non-equilibrium time evolution of the density matrix. The electrode degrees of freedom can be integrated out, and we obtain a reduced density matrix for the dot degrees of freedom only, labeled by $\chi$. The time evolution of the reduced density matrix, described by the propagator $\Pi_{\chi^{\prime} \chi}\left(t^{\prime}, t\right)$ for the propagation from a state $\chi$ at time $t$ to a state $\chi^{\prime}$ at time $t^{\prime}$, can be visualized by diagrams [2, 17] on the Keldysh contour. The full propagation is expressed as a sequence of irreducible blocks (self energies) $W_{\chi^{\prime} \chi}\left(t^{\prime}, t\right)$ that are associated with transitions from state $\chi$ at time $t$ to state $\chi^{\prime}$ at time $t^{\prime}$. This yields the Dyson equation

$$
\boldsymbol{\Pi}\left(t^{\prime}, t\right)=\mathbf{1}+\int_{t}^{t^{\prime}} d t_{2} \int_{t}^{t_{2}} d t_{1} \mathbf{W}\left(t_{2}, t_{1}\right) \mathbf{\Pi}\left(t_{1}, t\right)
$$

for the propagator, where the bold face indicates matrix notation related to the dot state labels. For the following, it is convenient to introduce the Laplace transform $\mathbf{W}(z)=\hbar \int_{-\infty}^{0} d t e^{z t} \mathbf{W}(0, t)$ and the definitions $\mathbf{W}=\left.\mathbf{W}(z)\right|_{z=0^{+}}$and $\partial \mathbf{W}=\left.(\partial \mathbf{W}(z) / \partial z)\right|_{z=0^{+}}$.

In the long-time limit, i.e. for time differences $t^{\prime}-t$ larger than the correlation time $(\propto 1 / \Gamma)$ over which the system forgets its initial state, the propagator becomes $\mathbf{p}^{\text {st }} \otimes \mathbf{e}^{T}$, where $\mathbf{e}^{T}=(1, \ldots, 1)$, and $\mathbf{p}^{\text {st }}$ is the vector of the stationary probabilities, determined from $\mathbf{W} \mathbf{p}^{\text {st }}=\mathbf{0}$, independent of the initial (diagonal) density matrix. As $\mathbf{W}$ has a zero eigenvalue, it cannot be inverted. With the normalization condition $\mathbf{e}^{T} \mathbf{p}^{\text {st }}=1$ we obtain the stationary probabilities $\mathbf{p}^{\text {st }}$ by solving

$$
\left(\tilde{\mathbf{W}} \mathbf{p}^{\mathrm{st}}\right)_{\chi}=\Gamma \delta_{\chi, \chi_{0}},
$$

where $\tilde{\mathbf{W}}$ is identical to $\mathbf{W}$ but with one (arbitrarily chosen) row $\chi_{0}$ being replaced by $(\Gamma, \ldots, \Gamma)[2]$. For a diagrammatic representation of the current, we introduce a block $\mathbf{W}^{I}$, in which one (internal) vertex is replaced by an external due to the current operator. We get

$$
I=\frac{e}{2 \hbar} \mathbf{e}^{T} \mathbf{W}^{I} \mathbf{p}^{\mathrm{st}} .
$$

The shot noise, Eq. (11), involves expectation values of two current operators. They either appear both in a single irreducible block, which we denote by $\mathbf{W}^{I I}$, or in two different blocks $\mathbf{W}^{I}$. We find

$$
S=\frac{e^{2}}{\hbar} \mathbf{e}^{T}\left[\mathbf{W}^{I I}+\mathbf{W}^{I}\left(\mathbf{P} \mathbf{W}^{I}+\mathbf{p}^{\text {st }} \otimes \mathbf{e}^{T} \partial \mathbf{W}^{I}\right)\right] \mathbf{p}^{\text {st }}
$$

with $\partial \mathbf{W}^{I}=\left.\left(\partial \mathbf{W}^{I}(z) / \partial z\right)\right|_{z=0^{+}}$. The object $\mathbf{P}=$ $\int_{-\infty}^{0} d t \frac{1}{\hbar}[\boldsymbol{\Pi}(0, t)-\boldsymbol{\Pi}(0,-\infty)]$ is determined by

$$
\tilde{\mathbf{W}} \mathbf{P}=\left(\mathbf{p}^{\text {st }} \otimes \mathbf{e}^{T}-\mathbf{1}\right)\left(\mathbf{1}-\delta_{\chi^{\prime}, \chi_{0}}\right)-\partial \tilde{\mathbf{W}} \mathbf{p}^{\text {st }} \otimes \mathbf{e}^{T} .
$$

Here we use the extra condition $\mathbf{e}^{T} \mathbf{P}=\mathbf{0}$, which follows from the definition of $\mathbf{P}$, the Dyson equation, and
$\mathbf{e}^{T} \mathbf{W}=\mathbf{0}$. The set of matrix equations Eqs. (3) - (6) constitute the starting point for all numerical results presented below. For a systematic perturbation expansion of current $I$ and noise $S$ in $\Gamma$, we expand all quantities $\mathbf{W}, \mathbf{W}^{I}, \mathbf{W}^{I I}, \partial \mathbf{W}, \partial \mathbf{W}^{I}, \mathbf{p}^{\text {st }}$ and $\mathbf{P}$ order by order. We remark that for transport in first order (sequential tunneling), the above expressions simplify, as all contributions involving the derivatives $\partial \mathbf{W}$ and $\partial \mathbf{W}^{I}$ disappear as a consequence of the fact that $\mathbf{W}$ starts at order $\Gamma$, $\mathbf{p}^{\text {st }}$ at $\Gamma^{0}$, and $\mathbf{P}$ at $\Gamma^{-1}$. These derivatives are associated with non-Markovian behavior of the system, and have not been taken into account in Refs. 3 and 2 . They are absent for first-order transport but are important for second- and higher-order corrections. (For a discussion of non-Markovian effects see also Ref. 18.) Higher derivatives will not appear for the shot noise even for higherorder corrections.

In the following we will focus on the Anderson impurity model, but we emphasize that the expressions presented above also cover more general situations, such as quantum dots or molecules with many levels and more general forms of the interactions [19, 20].

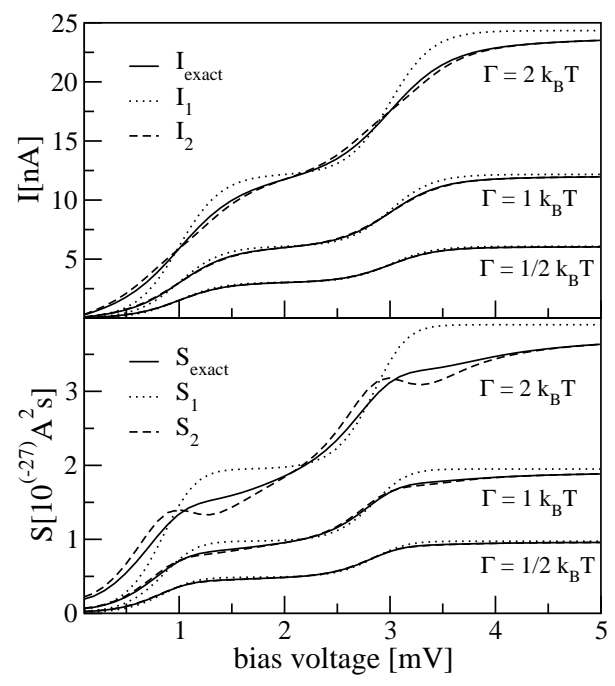

FIG. 1: Current $I$ and shot noise $S$ vs bias voltage for $k_{\mathrm{B}} T=$ $0.1 \mathrm{meV}, \epsilon_{\downarrow}=-1.5 \mathrm{meV}, \epsilon_{\uparrow}=0.5 \mathrm{meV}, U=0$, and $\Gamma_{\mathrm{L}}=\Gamma_{\mathrm{R}}=$ $\Gamma / 2$. First order (dotted lines) and second order (dashed lines) are compared to the exact results (solid lines) for $\Gamma / k_{\mathrm{B}} T=$ $0.5,1,2$.

Results. - We recall first the main features of first-order (sequential) transport. Both current and noise increase monotonically with bias voltage displaying plateaus separated by thermally broadened steps. The step positions are determined by the energy of singlecharge excitations. The coupling parameters set the plateau heights.

Higher-order processes modify the current and noise in two different ways. First, they increase the width of 
the steps, which effectively is given by the sum of $\Gamma$ and $T$. Second, they allow for transport in the Coulombblockade region at low bias, where sequential tunneling is suppressed. With increasing coupling strength $\Gamma$, secondand eventually also higher-order corrections to transport become more and more important. To illustrate the validity range of our second-order perturbation expansion we first consider the non-interacting limit, $U=0$, since in this case exact results [1, 9] are available for the current $I_{U=0}=e / h \int d \omega \sum_{\sigma} \tau_{\sigma}(\omega)\left(f_{\mathrm{L}}(\omega)-f_{\mathrm{R}}(\omega)\right)$ and shot noise $S_{U=0}=2 e^{2} / h \int d \omega \sum_{\sigma}\left\{\tau_{\sigma}(\omega)\left[f_{\mathrm{L}}(\omega)\left(1-f_{\mathrm{L}}(\omega)\right)+\right.\right.$ $\left.\left.f_{\mathrm{R}}(\omega)\left(1-f_{\mathrm{R}}(\omega)\right)\right]+\tau_{\sigma}(\omega)\left(1-\tau_{\sigma}(\omega)\right)\left[f_{\mathrm{L}}(\omega)-f_{\mathrm{R}}(\omega)\right]^{2}\right\}$

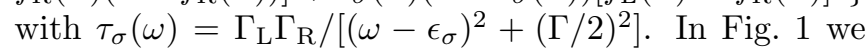
compare current and noise in first and second-order with the exact results. We choose a symmetric bias voltage $\left(\mu_{\mathrm{L}}=-\mu_{\mathrm{R}}=e V / 2\right)$. Outside the Coulomb-blockade regime, the second-order corrections (dashed lines) to sequential tunneling (dotted lines) start to become important for $\Gamma / k_{\mathrm{B}} T<0.5$. As long as $\Gamma / k_{\mathrm{B}} T<1$, the exact curves (solid lines) are perfectly reproduced by second-order perturbation theory, while the sequentialtunneling results clearly deviate. For $\Gamma / k_{\mathrm{B}} T=2$ thirdorder contributions start to play a role, at least for the noise, where unphysical non-monotonicities arise around the steps. We, therefore, restrict ourselves in the following discussion to $\Gamma \leq k_{\mathrm{B}} T$.

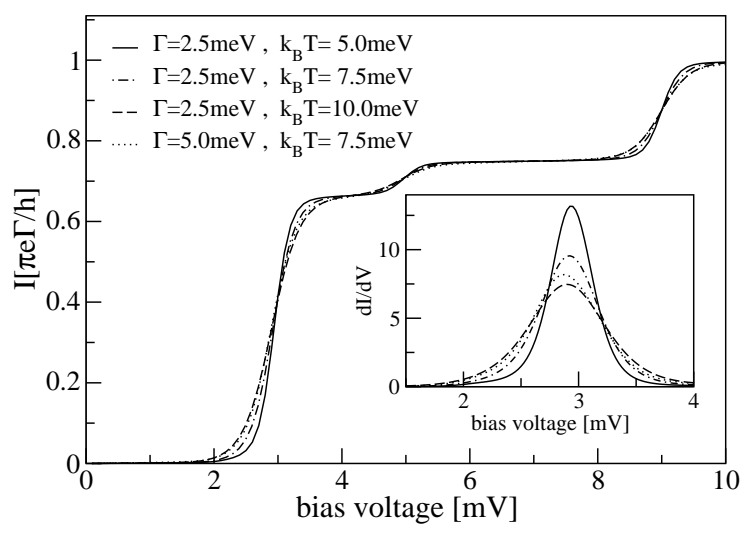

FIG. 2: Current $I$ and conductance $d I / d V$ (inset) vs biasvoltage for $\epsilon_{\downarrow}=-1.5 \mathrm{meV}, \epsilon_{\uparrow}=0.5 \mathrm{meV}, U=4 \mathrm{meV}$ and $\Gamma_{\mathrm{L}}=\Gamma_{\mathrm{R}}=\Gamma / 2$ for various values of $\Gamma$ and $k_{\mathrm{B}} T$. The broadening of the first step due to $\Gamma$ and $k_{\mathrm{B}} T$ is shown in the inset. The dashed and dotted curves with the same $\left(\Gamma+k_{\mathrm{B}} T\right)$ have about the same width.

The elastic co-tunneling processes which do not change the dot state or its energy allow for an electron exchange with the reservoirs via an intermediate virtual state. This leads to a finite linear conductance $G=d I /\left.d V\right|_{V=0}$. Also the noise persists at zero bias, $S=4 k_{\mathrm{B}} T G$, consistent with the equilibrium fluctuation-dissipation theorem (FDT). In the Coulomb blockade regime the FDT can be extended to non-equilibrium [16] and takes the form $S^{(2)}(V) / 2 e I^{(2)}(V)=\operatorname{coth}\left(e V / 2 k_{\mathrm{B}} T\right)$. Our theory satisfies this relation, however, we stress that it holds only in the regime of purely elastic co-tunneling processes. In Fig. 2 we show the current $I$ normalized to $\pi e \Gamma / h$ for the same set of energy parameters as in Fig. प but with a finite interaction $U=4 \mathrm{meV}$. Since the bias is applied symmetrically, the dot preferably occupies the state with spin $\downarrow$ (Coulomb-blockade) until it can be emptied due to first order hopping processes around $3 \mathrm{mV}$ (first step). Further steps arise around $5 \mathrm{mV}$ and $9 \mathrm{mV}$ due to the double occupied dot state. This parameter set is similar to the experimental situation of Ref. 15 for a quantum dot with occupation $N=2$ 21]. In Fig. 3 of that paper, a conductance step is observed inside the Coulomb blockade diamond, that is attributed to inelastic co-tunneling processes. For our model one expects this inelastic co-tunneling feature in the conductance at a bias of $\epsilon_{c o} / e=\left(\epsilon_{\uparrow}-\epsilon_{\downarrow}\right) / e=2 \mathrm{mV}$. This feature is hardly noticable in the conductance plot of the inset in Fig. 2] because the coupling $\Gamma$ is relatively weak and the energy $\epsilon_{c o}$ is fairly close to the sequential-tunneling energy. However, the inelastic co-tunneling processes can clearly be observed in the shot noise and the Fano factor $F=S / 2 e I$ discussed below.

We note that the dashed and dotted curves in Fig. 2 with same total sum $\left(\Gamma+k_{\mathrm{B}} T\right)$ almost lie on top of each other. The differential conductance plot (inset) shows that the temperature effect is a little stronger: the dashed curve with the highest temperature has the lowest peak. The FWHM of the conductance peaks is between $0.5 \mathrm{mV}-0.8 \mathrm{mV} \sim 6\left(\Gamma+k_{\mathrm{B}} T\right)$, as compared to $5.44 k_{\mathrm{B}} T$ for pure sequential tunneling [15]. We also note a shift of the peak position from the sequential-tunneling value $3 \mathrm{mV}$ to somewhat lower bias voltages, indicating a renormalization of the level positions.

The Fano factor $F=S / 2 e I$ for a fixed temperature and a sequence of coupling constants $\Gamma$, covering three orders of magnitudes, is shown in Fig. B At low bias, the Fano factor varies 16] as $\operatorname{coth}\left(e V / 2 k_{B} T\right)$ until it reaches the value 1 , as expected for uncorrelated systems. For bias voltages around the spin-flip excitation energy $\epsilon_{c o}=2 \mathrm{meV}$, the Fano factor becomes superPoissonian [16], $F>1$. Once sequential tunneling becomes dominant (at a bias $\geq 3 \mathrm{mV}$ ), it drops to values between 1 and $1 / 2$.

The super-Poissonian Fano factor appears for bias voltages at which the spin- $\uparrow$ level acquires some finite occupation probability, either due to inelastic spin-flip cotunneling, or due to sequential tunneling processes that are exponentially suppressed but, for the chosen parameters, still finite. The enhancement of the noise is mainly described by the second and third term of Eq. (5). It describes bunching of the transfered $\uparrow$-electrons during the time when this transport channel is not blocked by the dot being occupied with a $\downarrow$-electron. Spin flip pro- 


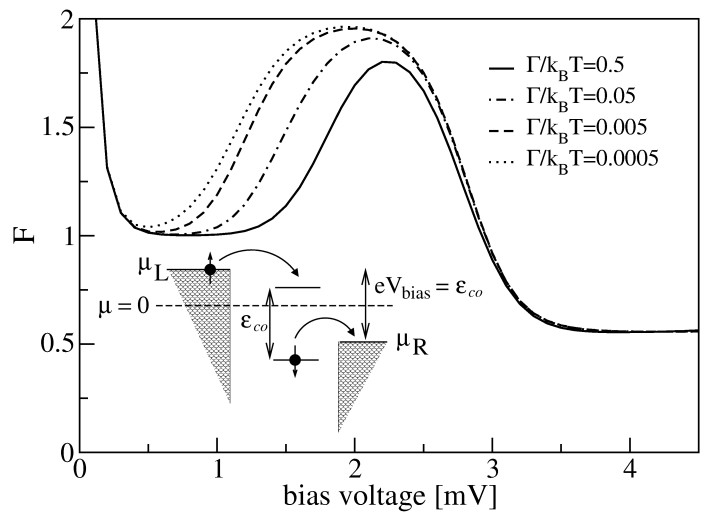

FIG. 3: Fano factor $F=S / 2 e I$ vs bias voltage for the same parameter set as Fig. 2 but fixed temperature $k_{\mathrm{B}} T=0.1 \mathrm{meV}$ and various $\Gamma$. Inelastic co-tunneling leads to a superPoissonian Fano factor if the bias is larger than $\epsilon_{c o} / e=2 \mathrm{mV}$. First order processes may also lead to a super-Poissonian value at a scale $2 \epsilon_{\uparrow} / e=1 \mathrm{mV}$. The crossover between these energy scales runs over three orders of magnitude in the coupling. Outside the Coulomb blockade regime the first order results are recovered already at $\Gamma / k_{\mathrm{B}} T \sim 0.1$. The inset shows a sketch of the transport situation at $e V_{\text {bias }}=\epsilon_{c o}$.

cesses leading to super-Poissonian noise have also been discussed in Ref. 7 in the case of ferromagnetic leads. Both the position and the height of the peak in the Fano factor depends on all system parameters. In Fig. 3 we study the dependence on the ratio $\Gamma / k_{\mathrm{B}} T$.

With reduced coupling strength $\Gamma$, the peak increases and moves towards lower bias. For $\Gamma / k_{\mathrm{B}} T=0.0005$ our result (dotted line) coincides with that of a pure first-order calculation. At larger coupling, though, the second-order terms lead to a severe modification of the result because the rates in the Coulomb-blockade regime depend only algebraically and not exponentially on the energy. We emphasize that, since the peak is close to the onset of sequential tunneling, an analysis purely based on co-tunneling processes [16 would not be sufficient either. The range of $\Gamma / k_{\mathrm{B}} T$ ratio over which second-order dominated co-tunneling crosses over to sequential tunneling is rather large, i.e., the Fano factor provides a sensitive measure of the coupling strength. For some range of the ratio $\Gamma / k_{\mathrm{B}} T$ the inelastic co-tunneling effects on the shot noise can be measured experimentally 22. The importance of the second-order processes for the peak of the Fano factor contrasts with the situation at larger bias beyond the sequential tunneling threshold when secondorder corrections only become noticable for $\Gamma / k_{\mathrm{B}} T \sim 0.1$.

In summary, we presented a theory of current and shot noise within a diagrammatic technique that includes higher-order tunneling processes in the coupling of a quantum dot to metallic electrodes. As an example, we studied current and noise for an Anderson impurity model with finite spin splitting. We showed that especially the steps and the Coulomb-blockade regions are strongly affected by second-order processes and provide additional information compared to first order. Spinflip processes of different origin in first and second order lead to super-Poissonian noise in the Coulomb-blockade regime. This provides a spectroscopic tool to characterize the dot-electrode couplings by a combined measurement of current and shot noise.

Acknowledgments. We profitted from discussions with J. Aghassi, A. Braggio, R. Fazio, G. Johansson, and J. Martinek, and acknowledge financial support by the DFG via the Center for Functional Nanostructures, SFB 491 and GRK 726.

[1] Ya. M. Blanter and M. Büttiker, Phys. Rep. 336, 1 (2000).

[2] A. Thielmann, M. H. Hettler, J. König, and G. Schön, Phys. Rev. B 68, 115105 (2003).

[3] S. Hershfield et al., Phys. Rev. B 47, 1967 (1993); A. N. Korotkov, Phys. Rev. B 49, 10381 (1993); Yu. V. Nazarov and J. J. R. Struben, Phys. Rev. B 53, 15466 (1996); G. Kiesslich et al., phys. stat. sol. (c) 0, 1293 (2003).

[4] G. Michalek and B. R. Bulka, Eur. Phys. J. B 28, 121 (2002).

[5] A. Thielmann, M. H. Hettler, J. König, and G. Schön, accepted by Phys. Rev. B, cond-mat/0406647.

[6] A. Mitra, I. Aleiner, and A. J. Millis, Phys. Rev. B 69, 245302 (2004).

[7] B.R. Bulka, J. Martinek, G. Michalek and J. Barnaś, Phys. Rev. B 60, 12246 (1999); A. Cottet, W. Belzig, and C. Bruder, Phys. Rev. Lett. 92, 206801 (2004).

[8] J. X. Zhu and A. V. Balatsky, Phys. Rev. B 67, 165326 (2003).

[9] S. Hershfield, Phys. Rev. B 46, 7061 (1992).

[10] M. Hamasaki, Phys. Rev. B 69, 115313 (2004).

[11] H. Birk, M. J. M. de Jong and C. Schönenberger, Phys. Rev. Lett. 75, 1610 (1995).

[12] S. S. Safonov et al., Phys. Rev. Lett. 91, 136801 (2003).

[13] A. Nauen et al., Phys. Rev. B 70, 033305 (2004).

[14] D.V. Averin and A.A. Odintsov, Phys. Lett. A 140, 251 (1989); D.V. Averin and Yu. V. Nazarov, Phys. Rev. Lett. 65, 2446 (1990).

[15] S. De Franceschi et al., Phys. Rev. Lett. 86, 878 (2001).

[16] E. V. Sukhorukov, G. Burkard and D. Loss, Phys. Rev. B 63, 125315 (2001).

[17] J. König, H. Schoeller, and G. Schön, Phys. Rev. Lett. 76, 1715 (1996); J. König, J. Schmid, H. Schoeller, and G. Schön, Phys. Rev. B 54, 16820 (1996).

[18] A. Braggio, J. König, and R. Fazio, in preparation (2004).

[19] M. H. Hettler, W. Wenzel, M. R. Wegewijs, and H. Schoeller, Phys. Rev. Lett. 90, 076805 (2003).

[20] In systems with degeneracies not related to symmetries of the Hamiltonian, a generalization of our approach to off-diagonal density matrices might be necessary.

[21] This is because the spin-split single impurity Anderson model can be mapped onto a two-level Anderson model[4, 5, 7] for the temperature and bias range of interest.

[22] In Ref. 13 shot noise of size $\sim 10^{-28} A^{2} s$ was measured. 